\title{
Characterization of mechanical properties of barium titanate ceramics with different grain sizes
}

\author{
TOMASZ TRZEPIECINSKI ${ }^{1, *}$, MAgDALENA GROMADA $^{2}$ \\ ${ }^{1}$ Rzeszow University of Technology, Department of Materials Forming and Processing, \\ Al. Powst. Warszawy 8, 35-959 Rzeszów, Poland \\ ${ }^{2}$ Institute of Power Engineering, Ceramic Department CEREL, ul. Techniczna 1, 36-040 Boguchwała, Poland
}

\begin{abstract}
In this paper, three $\mathrm{BaTiO}_{3}$ powders of various particle size distributions were obtained as a result of mechanical activation in the mixer mill. Green barium titanate pellets and cylindrical specimens were fabricated by both uniaxial and isostatic pressing methods. As a result of the application of different maximal sintering temperatures, the obtained materials were characterized by various average grain sizes: $0.8 \mu \mathrm{m}, 20 \mu \mathrm{m}$ and $31.0 \mu \mathrm{m}$. The basic properties of sintered pellets and cylinders were determined and the influence of materials average grain size on their Young's modulus and compressive strength were determined through compression tests in a uniaxial testing machine, Zwick/Roell Z100. The elastic properties were similar for tested materials with a different grain size. However, the microstructure of $\mathrm{BaTiO}_{3}$ strongly influenced the compressive strength.
\end{abstract}

Keywords: barium titanate; compression strength; mechanical properties, particle size distribution

\section{Introduction}

Piezoelectric ceramics are used extensively in many engineering applications because of their excellent dielectric characteristics. The ceramic materials are used in the manufacture of many engineering devices, for example, multilayer actuators (MLAs), multilayer ceramic capacitors (MLCCs), thermistors, piezoelectric transducers and emerging MEMS technologies. In the piezoelectric multilayer actuator design, it is desirable to account for unpoled material that is not piezoelectric and possesses different permittivity and elastic constants to those of the poled state [1].

A high capacitance of small-sized MLCCs requires a large number of ceramic layers with a small thickness. This can cause mechanical failure owing to the difference in density between the electrode and the margin layer without electrode [2]. The assessment of mechanical properties of piezoelectric ceramics can help to understand the development of residual stresses within the ceramic layers and improve the reliability and the lifetime of

*E-mail: tomtrz@prz.edu.pl
MLAs. To measure the residual (internal) stresses and mechanical properties of the perovskite materials, several methods are used, i.e. bending test, compression test or sharp indentation method [3]. Because of the inherently brittle nature of ceramics, its strength is invariably controlled by the largest pore in the microstructure [4]. The morphology of the powders consists of particles and their agglomerates, whose dimensions depend on the synthesis method [5]. If the size and/or shape of the components are altered, the effect of the largest pore will also be subject to a change [6]. To produce a barium titanate ceramics with the mechanical strength that is highly repeatable between components of a batch, requires low porosity arising from small spherical pores spread uniformly throughout the material [7]. This criterion also leads to the higher mechanical strength of the ceramics.

Barium titanate $\left(\mathrm{BaTiO}_{3}\right)$ is one of the most widely used functional materials in electric ceramics. In general, $\mathrm{BaTiO}_{3}$ is isotropic in the unpoled state and is characterized by two elastic constants: Poisson ratio and Young modulus. $\mathrm{BaTiO}_{3}$ exhibits a complex phase appearance, and between $120{ }^{\circ} \mathrm{C}$ and $1457{ }^{\circ} \mathrm{C}$ it reveals a cubic perovskite 
structure that consists of corner-linked octahedral oxygen containing $\mathrm{Ti}^{4+}$, with $\mathrm{Ba}^{2+}$ [8]. In the temperature interval between $5{ }^{\circ} \mathrm{C}$ and $120^{\circ} \mathrm{C}, \mathrm{BaTiO}_{3}$ has a tetragonal polar ferroelectric phase [9]. The applicability of $\mathrm{BaTiO}_{3}$ piezoelectric material is limited to the temperature range of stability of the polar ferroelectric phase; i.e. between approximately $5{ }^{\circ} \mathrm{C}$ (the tetragonal-to-orthorhombic transition) and $120{ }^{\circ} \mathrm{C}$ (the cubic-to-tetragonal transition) [8]. The frequency response of resonant devices may be disturbed by differences in the elastic constants of piezoelectric ceramics. The properties of electronic ceramics are also influenced by the morphology of material and particle size $[10,11]$. Grain size strongly affects the dielectric properties of $\mathrm{BaTiO}_{3}$. At room temperature, the dielectric constant for coarse-grained ceramics is lower than for fine-grained ones [12]. However, if the grain size is too small then the dielectric constant decreases again to lower values [10]. In order to achieve an optimum value of the grain size in fired barium titanate, grain-growth inhibitors can be used. Another way to control the grain growth is the strict time-temperature control of the sintering process.

Several synthesis routes and sintering regimes can be used to obtain the $\mathrm{BaTiO}_{3}$ nanopowders; i.e. solid-state or sol-gel technique, hydrothermal synthesis, chemical co-precipitation, mechanochemical activation or mechanical synthesis. Vijatović et al. [13] concluded that the synthesis method has a great effect on the characteristics of powders and ceramics, influencing the end application. In this paper, barium titanate powder has been manufactured using a traditional solid-state routine because this method allows precise stoichiometric control. Furthermore, this method guarantees acceptable results at the lowest cost. This paper is concerned with the effect of the grain size of barium titanate on its mechanical properties. The main parameters of barium titanate powders (particle size distribution) and sinters (apparent density, water absorbability, apparent porosity, average grain size) were determined and fractography analysis was performed. The basic mechanical properties of the cylindrical specimens were estimated through a uniaxial compression test.

\section{Experimental}

\subsection{Powder preparation}

The powder of barium titanate perovskite was fabricated by the solid-state method and then granulated in order to improve its mouldability. The detailed description of powder preparation, evaluation of its properties and granulation process was presented in our previous paper [14]. The obtained powder and granulate were treated as the basis and denoted as powder and granulate a.

Pellets and cylindrical specimens for the compression test were obtained in a mould with an external diameter of $11.5 \mathrm{~mm}$, where $\mathrm{BaTiO}_{3}$ granulate a with a weight of $0.3 \mathrm{~g}$ and $12 \mathrm{~g}$, for pellets and cylindrical specimens, respectively, underwent uniaxial pressing under a pressure of $1 \mathrm{MPa}$ and $10 \mathrm{MPa}$, correspondingly. The green pellets and cylinders obtained in this manner were then pressed isostatically under a pressure of $150 \mathrm{MPa}$, and sintered in the electric furnace in an air atmosphere according to the sintering curve, ensuring high density of obtained sinters. The maximal sintering temperature was $1250{ }^{\circ} \mathrm{C}$, the rate of both heating and cooling was $100{ }^{\circ} \mathrm{C} / \mathrm{h}$, whereas the dwell time was $4 \mathrm{~h}$. The obtained pellets and cylinders were denoted as BT_a.

\subsection{Grain size}

In order to obtain two additional different grain size distributions of the finer course, the barium titanate powder was activated mechanically in a mixer mill in two lots for 3 hours and 5 hours. These powders took the following denotation: powder_b and powder_c, for the milling for 3 and 5 hours, respectively. Pellets and cylindrical specimens from granulate $b$ and $c$ were manufactured according to the same procedure described above. In order to obtain a high density simultaneously with ensuring different grain size of the sinters, pellets and cylinders made from the granulates $b$ and $\mathrm{c}$, they were sintered at the maximal temperatures equal to $1250{ }^{\circ} \mathrm{C}$ and $1350{ }^{\circ} \mathrm{C}$, respectively. The rate of both heating and cooling was $100{ }^{\circ} \mathrm{C} \cdot \mathrm{h}^{-1}$, whereas the dwell time was $4 \mathrm{~h}$. These pallets and cylinders took the following denotation BT_b and 
BT_c, for pellets and cylinders, respectively, fabricated from granulates $b$ and $c$. The cylindrical specimens for the compression test of all materials were ground using a grinding machine with a diameter equal to $9.0 \mathrm{~mm}$ and height $13.5 \mathrm{~mm}$.

The grain size distributions of barium titanate powders were determined using Mastersize 2000 from the Melvern Company. Parameters characterizing the extent of sintering of the barium titanate materials - apparent density, apparent porosity and water absorbability - were estimated using Archimedes law. In order to determine the average grain size of $\mathrm{BaTiO}_{3}$ sintered materials, the microstructure was evaluated by means of scanning electron microscopy (SEM) HITACHI S-3400N/2007 and then the secant method was applied. The size and shape of pores in sintered materials were determined on the basis of analysis of SEM photos of fracture surfaces.

\subsection{Compression test}

The compression tests were carried out using a universal uniaxial testing machine Zwick/Roell Z100. The compression tests were performed at a room temperature and four specimens for each cylindrical specimen variant were tested to evaluate the average values of Young modulus $\mathrm{E}$ and compression strength $\mathrm{R}_{\mathrm{c}}$. The compression test of $\mathrm{BaTiO}_{3}$ ceramics was carried out on the cylindrical specimens with a diameter of $9 \mathrm{~mm}$ and a height of $13.5 \mathrm{~mm}$.

\section{Results and discussion}

\subsection{Particle size distribution}

The particle size distributions of all barium titanate powders presented in Fig. 1 are unimodal and quite narrow. The mean particle size of basic $\mathrm{BaTiO}_{3}$ powder a is equal to $1.98 \mu \mathrm{m}$, whereas the $\mathrm{d}_{10}$ and $\mathrm{d}_{90}$ are equal to $0.99 \mu \mathrm{m}$ and $3.72 \mu \mathrm{m}$, respectively. The mechanical activation of barium titanate powder a for 3 hours and 5 hours caused a decrease in the mean grain size to $1.20 \mu \mathrm{m}$ and $0.58 \mu \mathrm{m}$, respectively, for powders $\mathrm{b}$ and $\mathrm{c}$.

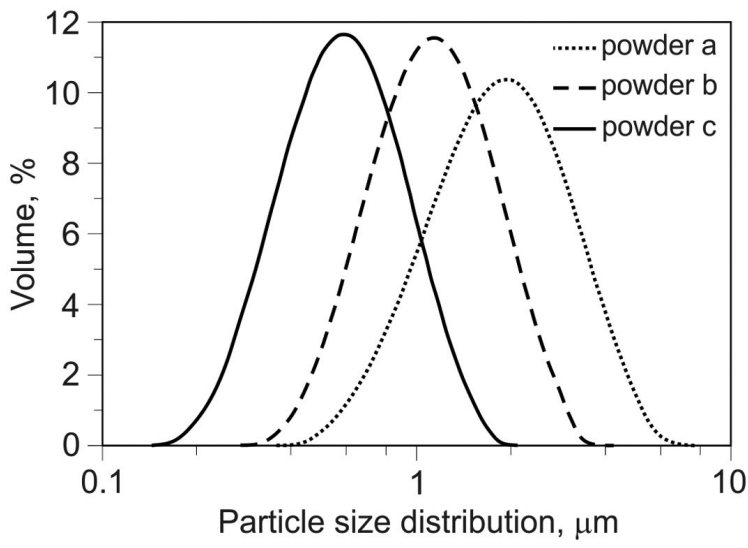

Fig. 1. Particle size distribution of $\mathrm{BaTiO}_{3}$ powder $\mathrm{a}, \mathrm{b}$ and $\mathrm{c}$.

Table 1 presents physical properties of sintered barium titanate materials. As a result of pellets sintering made from the three granulates $a, b$ and c of different grain-size distributions at various temperatures $\left(1250{ }^{\circ} \mathrm{C}\right.$ and $\left.1350{ }^{\circ} \mathrm{C}\right)$, very similar apparent densities were obtained, whereas the average grain diameters differed from $0.8 \mu \mathrm{m}$ to $31.0 \mu \mathrm{m}$, which is in accordance with the former expectation.

The theoretical density of the sintered $\mathrm{BaTiO}_{3}$ was assumed to be equal $6.02 \mathrm{~g} / \mathrm{cm}^{3}$, based on the lattice constant value [15]. The obtained relative densities for all pellets presented in Table 1 are very high and comparable to those presented by Duran et al. [15]. All pellets reveal quite low apparent porosity and almost negligible water absorbability.

During sintering, the grains grow and the grain shape may also be changed. The increase in the specific surface area of the particles by decreasing the particle size of the raw material may accelerate the sintering process. This can be explained by the fact that the driving force of sintering is related to the surface energy of particles [16].

\subsection{Compressive strength}

The machine used to provide the compression loading was a Zwick/Roell uniaxial testing machine. Loading speed was $2 \mathrm{~mm} / \mathrm{min}$ [4]. The biggest problem in ceramics testing under 
Table 1. The physical properties of $\mathrm{BaTiO}_{3}$ materials after sintering.

\begin{tabular}{lccc}
\hline \multirow{2}{*}{ Parameter } & \multicolumn{3}{c}{ Sample notation } \\
\cline { 2 - 4 } & BT_a & BT_b & BT_c \\
\hline \hline Apparent density $\left[\mathrm{g} / \mathrm{cm}^{3}\right]$ & 5.84 & 5.85 & 5.86 \\
Relative density $[\%]$ & 97.0 & 97.2 & 97.3 \\
Apparent porosity [\%] & 0.19 & 0.10 & 0.16 \\
Water absorbability $[\%]$ & 0.03 & 0.02 & 0.03 \\
Average grain diameter $[\mu \mathrm{m}]$ & 31.0 & 0.8 & 20.0 \\
\hline
\end{tabular}

compression loading is prevention of local stress concentration, owing to the non-uniform contact of the sample surface to the load surface. Hence, a small range of tolerance of the parallelism of the specimen faces and their perpendicularity to the specimen axes is required. High demands are also imposed on the surface finish of the sample front surfaces. These surfaces cannot exhibit any cracks, crevices and gaps, and should be ground. The pellets were examined prior to testing and positioned in such a way as to eliminate erroneous results by using an obviously damaged region as one of the loaded contacts.

All of the $\mathrm{BaTiO}_{3}$ cylindrical specimens responded linearly to the compressive loading up to failure. The values of Young modulus and the compressive strength of barium titanate ceramics are presented in Table 2. The value of Young modulus is similar for the tested materials with different grain sizes. However, the microstructure of $\mathrm{BaTiO}_{3}$ strongly influences the compressive strength $R_{c}$. The highest value of $R_{c}$ was obtained for sample BT_b, revealing the smallest grain size, which is in accordance with earlier expectation. The second value of compressive strength was obtained for material BT_a, revealing the highest grain size, whereas the lowest strength was exhibited by material BT_c with an average grain size equal to $20.0 \mu \mathrm{m}$. This result can be caused by material densification of BT_c, so there is a large amount of small closed pores in the microstructure.

The compression strength of ceramics is between 5 and 10 times greater than the bending strength as indicated in Brevier Technical Ceramics [17]. Furthermore, the compressive strength of the ceramic materials is 5 to 10 times greater than the tensile strength [18]. So, ceramics, particularly $\mathrm{BaTiO}_{3}$, are much stronger in compression than in tension. Large differences between tensile and compressive strength lead to poor shock resistance. Lack of homogeneities in the microstructure and surface finish play a less important role here. Where possible, therefore, ceramics should be subject to compressive stress.

\subsection{Fractography analysis}

Fractography examinations are an important tool used to identify fracture mode and identify features in the fracture process. The micrographs analyses of fracture surfaces (Fig. 2), made by scanning electron microscope, indicate that there is a mixture of transgranular and intergranular modes of fracture. The fracture surface of $\mathrm{BaTiO}_{3}$ ceramics in macro scale (Fig. 2) subjected to compression stress is significantly different from the fracture surface formed at different stress states. The fracture surface of all types of tested pellets is characterized by shear fracture mode with visible leap branching. The fracture surfaces of materials that fail in a brittle manner from surface cracks are characterized by a sequence of three distinct fracture features (mirror, mist, hackle) surrounding the critical crack which initiated the fracture [19]. The hackle markings seen on the fracture surface (Fig. 2a) are distinct from those observed on the failed fracture surfaces in a combination of tensile and shear loading conditions, where hackle markings appear as lances. The hackle markings at the start of crack propagation, when a portion of the crack begins propagating out of the crack plane, were also observed by Gopalakrishnan et al. [19]. 
Table 2. The mechanical properties of $\mathrm{BaTiO}_{3}$ materials after sintering.

\begin{tabular}{llll}
\hline \multirow{2}{*}{\multicolumn{1}{c}{ Parameter }} & \multicolumn{3}{c}{ Sample notation } \\
\cline { 2 - 4 } & BT_a & BT_b & BT_c \\
\hline \hline Young modulus E [GPa] & 115.5 & 118.0 & 116.0 \\
Compressive strength $\mathrm{R}_{\mathrm{c}}[\mathrm{MPa}]$ & 655.7 & 913.2 & 486.2 \\
\hline
\end{tabular}
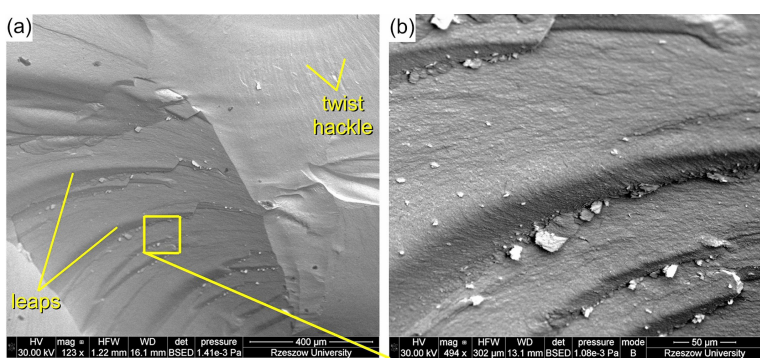

Fig. 2. SEM microphotographs of microstructure of BT_a in BSE mode at different magnifications: (a) $\times 123$; and (b) $\times 494$.

Fig. 3 presents the fracture surface of all materials after sintering and the execution of the compression test. Observations revealed the transgranular propagation of the cracks. It can be seen from the SEM micrographs that there is a significant difference in the microstructure between the BT_a and BT_b cylindrical specimens. For cylinders BT_a and BT_c, which possess an average grain size equal to $31.0 \mu \mathrm{m}$ and $20.0 \mu \mathrm{m}$. respectively, the estimated values of pores diameter have almost the same size and shape of pores, which, in these two cases, are spherical. However, for the sample BT_b, revealing considerable smaller grain size $(0.8 \mu \mathrm{m})$, the pores also have lower size and possess an oblong shape. As expected, the highest compressive strength of BT_b corresponds to the smaller grain size.

As failure of ceramics usually initiates from pores or cracks within the component, porosity is a very important quantity. To ensure a long service life, porosity should be kept to a minimum. Pores can be both closed and open. They weaken the material, but if their shape is rounded their effect on both crack initiation and propagation is less detrimental. The microcracks which can be considered as pores with sharp edges exhibit a more
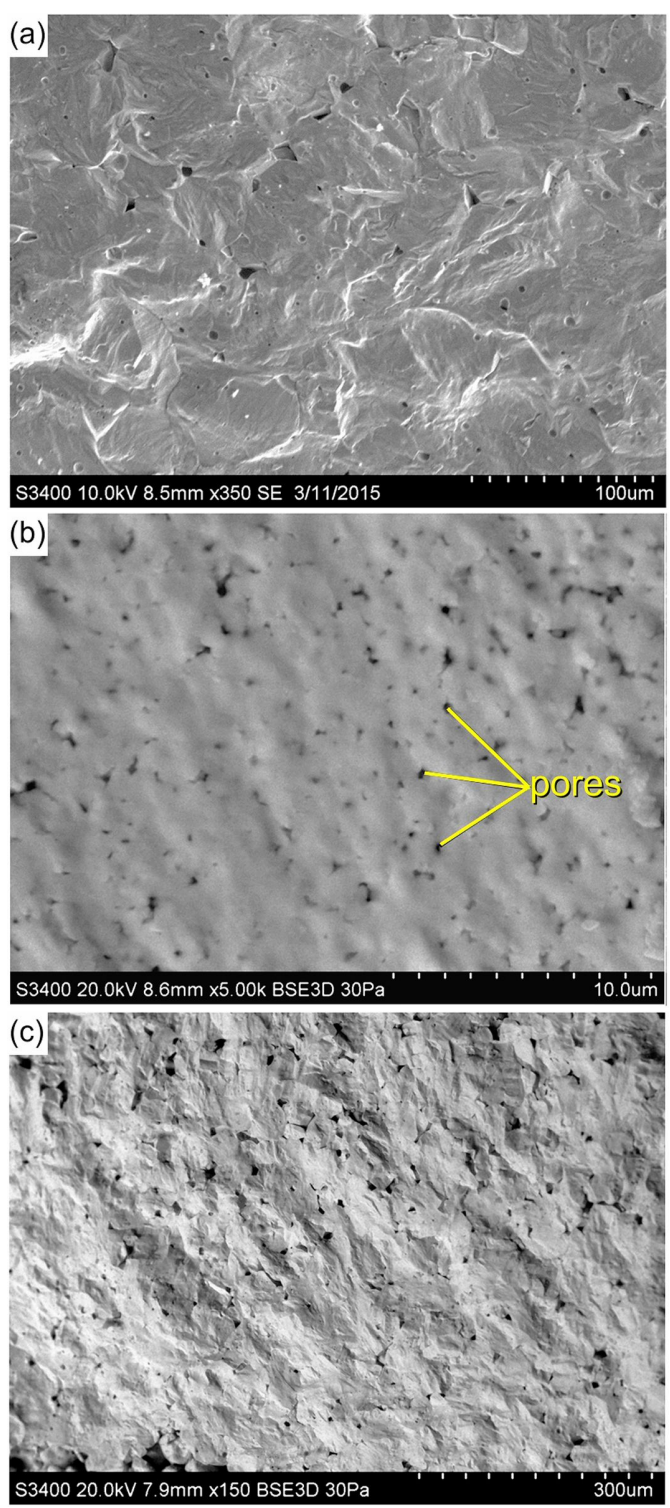

Fig. 3. Cylinders fracture surface of: (a) BT_a (mag. $\times 350$, SE mode); (b) BT_b (mag. $\times 5000$, BSE mode); and (c) BT_c (mag. $\times 150$, BSE mode).

harmful effect on ceramics failure than pores. Microcracks are generated in the fabrication 
process and are a result of differences in linear expansion coefficients or elastic moduli of individual anisotropic grains or ceramic phase components. Both microcracks and porosity determine the strength of the ceramic materials.

\section{Summary and conclusions}

Barium titanate ceramics are required to withstand not only mechanical loading but also thermal stresses. The brittle nature of the ceramics depends mainly on the porosity of the material. Barium titanate, being a granular aggregate, has pore sites between the grains which may act as fracture initiators. Failure in barium titanate ceramics appears to be initiated by large pores, a series of smaller pores, or microcracks which can be a result of the fabrication process. The microstructure of $\mathrm{BaTiO}_{3}$ strongly influences the compressive strength. It was found that the compressive strength depends on the grain size and the shape of the grains. A higher compressive strength was found for barium titanate with an average oblongshaped grain size of $0.8 \mu \mathrm{m}$. The elastic properties were similar for tested materials with a different grain size.

The literature review indicates that ceramic materials subjected to tensile stress are destroyed at very low stresses. This is owing to the presence in the ceramics of defects in the form of cracks and voids that open out under tension. Compressive stresses 'close' these defects and therefore the compressive strength of ceramics is 5 to 10 times greater than the tensile strength.

\section{Acknowledgements}

The research leading to these results has received funding from the People Programme (Marie Curie Actions) of the European Union's Seventh Framework Programme FP7/20072013/ under the REA Grant Agreement No. PITN-GA-2013606878. The authors of this paper would like to kindly thank Dr. Małgorzata Wierzbińska from Rzeszow University of Technology and Dr. Wojciech Bochnowski from the University of Rzeszów for the preparation of the SEM micrographs.

\section{References}

[1] Dent A.C., Bowen C.R., Stevens R., Cain M.G., Stewart M., J. Eur. Ceram. Soc., 27 (2007), 3739.

[2] Shin Y.I., KAng K.M., Jung Y.G., Yeo J.G., LeE S.G., PAIK U., J. Eur. Ceram. Soc., 23 (2003), 1427.

[3] RyU S.S., Kim H.T., Kim H.J., Kim S., J. Ceram. Soc. Jpn., 117 (2009), 811.

[4] BLAMEY J.M., Investigation into the strength, toughness and porosity of barium titanate PTC ceramics, Durham University, Durham, 1990.

[5] Lazarević Z., Romčević N., ViJatović M., Paunović N., RomČEvić M., STOJAnović B., DohČević-Mitrović Z., Acta Phys. Pol. A, 115 (2009), 808.

[6] Almond E.A., Roebuck B., Gee M.G., Inst. Phys. Conf. Ser., 75 (1986), 155.

[7] Blamey J.M., Parry T.V., J. Mater. Sci., 28 (1993), 4988.

[8] Ctibor P., Seiner H., Sedlacek J., Pala Z., VANEK P., Ceram. Int., 39 (2013), 5039.

[9] Zhao M.H., Bonnell D.A., Vohs J.M., Surf. Sci., 602 (2008), 2849.

[10] Miclea C., Tănăsoiu C., SpĂnulescu I., MiClea C.F., GheorghiU A., Amarande L., Cioangher M., Miclea C.T., Rom. J. Inf. Sci. Tech., 10 (2007), 335.

[11] Prasad V.C.S., Kumar L.G.K., Ferroelectrics, 102 (1990), 141.

[12] Arlt G., Penses H., Ferroelectrics, 48 (1983), 213.

[13] Vijatović M.M., Bobić J.D., Stojanović B.D., Sci. Sinter., 40 (2008), 155.

[14] Gromada M., Biglar M., Trzepieciński T., StaCHOWICZ F., B. Mater. Sci., 40 (2017), 759.

[15] Duran P., Gutierrez D., Tartaj J., Moure C., Ceram. Int., 28 (2002), 283.

[16] UCHINo K., Advanced piezoelectric materials, science and technology, Woodhead Publishing, Duxford, 2017.

[17] Verband der Keramischen Industrie e.V., Brevier technical ceramics, Verlag Hans Fahner GmbH \& Co. KG, Lauf, 2003.

[18] Mittal J.P., Kaur I., Sharma R.C., Encyclopedia of technical education - industrial engineering and materials, Mittal Publications, New Delhi, 1992.

[19] Gopalakrishnan K., Mecholsky J.J., J. Eur. Ceram. Soc., 34 (2014), 3247. 